\title{
Aggressive Delinquency Among North American Indigenous Adolescents: Trajectories and Predictors
}

\author{
Kelley J. Sittner ${ }^{1 *}$ and Dane Hautala ${ }^{2}$ \\ ${ }^{1}$ Department of Sociology, Oklahoma State University, Stillwater, Oklahoma \\ ${ }^{2}$ Department of Sociology, University of Nebraska-Lincoln, Lincoln, Nebraska
}

: : : : : : : : : : : : : : : : : : : : : : : : : : : : : : : : : : :

Aggressive delinquency is a salient social problem for many North American Indigenous (American Indian, Canadian First Nations) communities, and can have deleterious consequences later in life. Yet there is a paucity of research on Indigenous delinquency. Group-based trajectory modeling is used to prospectively examine trajectories of aggressive delinquency over the course of adolescence using data from 646 Indigenous adolescents from a single culture, spanning the ages of 10-19. Five aggression trajectory groups were identified, characterized by different levels and ages of onset and desistence: non-offenders (22.1\%), moderate desistors (19.9\%), adolescent-limited offenders $(\mathbf{2 2 . 2 \% )}$, high desistors $(\mathbf{1 6 . 7 \% )}$, and chronic offenders $(\mathbf{1 9 . 2 \%})$. Using the social development model of antisocial behavior, we selected relevant risk and protective factors predicted to discriminate among those most and least likely to engage in more aggressive behavior. Higher levels of risk (i.e., parent rejection, delinquent peers, substance use, and early dating) in early adolescence were associated with being in the two groups with the highest levels of aggressive delinquency. Positive school adjustment, the only significant protective factor, was associated with being in the lowest aggression trajectory groups. The results provide important information that could be used in developing prevention and intervention programs, particularly regarding vulnerable ages as well as malleable risk factors. Identifying those youth most at risk of engaging in higher levels of aggression may be key to preventing delinquency and reducing the overrepresentation of Indigenous youth in the justice system. Aggr. Behav. 42:274-286, 2016. () 2015 Wiley Periodicals, Inc.

Keywords: aggression; delinquency; American Indian; trajectories

\section{INTRODUCTION}

Aggressive delinquency is a salient social problem for many North American Indigenous (American Indian, Canadian First Nations) communities. Compared to other ethno-cultural groups, rates of violent victimization, homicide, and suicide are higher for Indigenous people on reservations and reserves (Bachman, 1992; Royal Commission on Aboriginal Peoples, 1996). Additionally, certain types of health risk behavior may be higher for Indigenous youth. For instance, $17.8 \%$ of the American Indian youth in the Youth Risk Behavior Surveillance System study reported carrying a weapon in the past month compared to $12.5 \%$ of African American youth, and the American Indian youth were more than two times as likely as African American youth to be threatened with a weapon at school (Centers for Disease Control and Prevention, 2013). Indigenous youth are also victims of violent crimes at rates far exceeding other groups (Perry, 2004; Statistics Canada, 2001).

This exposure to and involvement in aggressive behavior can have deleterious effects later in life. For example, it may place youth at greater risk of justice system involvement. In 2010, over $40 \%$ of youth in custody were there for violent offenses (Sedlak \& Bruce, 2010). Involvement in the justice system can have cascading effects on later well being, including impaired educational attainment (Sweeten, 2006) and violent offending (DeLisi, Hochstetler, Johnson, Caudill, \& Marquart, 2011). Furthermore, there is considerable continuity of aggressive behavior from childhood and adolescence through adulthood (Loeber, Farrington, \&

Contract grant sponsor: National Institute on Drug Abuse; contract grant number: DA13580; contract grant sponsor: National Institute of Mental Health; contract grant number: MH67281; contract grant sponsor: National Institute of Alcoholism and Alcohol Abuse; contract grant number: AA020299.

*Correspondence to: Kelley J. Sittner, Department of Sociology, Oklahoma State University, 431 Murray Hall, Stillwater, OK 74078. E-mail: kelley.sittner@okstate.edu

Received 24 January 2015; Revised 8 July 2015; Accepted 14 July 2015

DOI: $10.1002 / \mathrm{ab} .21622$

Published online 9 September 2015 in Wiley Online Library (wileyonlinelibrary.com). 
Waschbusch, 1998). According to Duran and Duran (1995), one in three Indigenous male adults in the United States will be incarcerated at some point in their lives. Given the prevalence of violence experienced by Indigenous people, as well as the linkages between early aggression and later adult criminality, it is important that we understand who is likely to be involved in aggressive behavior at younger ages and over longer periods of time. Yet there is little research on Indigenous offending in general (Pridemore, 2004), and delinquency in particular.

This is the first study to prospectively examine trajectories of aggressive delinquency over the course of adolescence among a sample of Indigenous youth and to identify correlates that differentiate membership in the different trajectory groups. We apply the social development model of antisocial behavior to identify important risk and protective factors in childhood/early adolescence that may explain differential involvement in aggressive behavior. Those risk and protective factors are then used to create profiles of the trajectory groups and to predict trajectory group membership. We begin by reviewing the research on delinquency trajectories in general and with Indigenous samples in particular.

\section{RESEARCH ON DELINQUENCY TRAJECTORIES}

Involvement in criminal behavior increases in early adolescence, peaks around ages 16-18, and decreases thereafter, a pattern often referred to as the age-crime curve (Blumstein, Cohen, \& Farrington, 1988). This increase in delinquent behavior has been attributed to changes both in the number of juveniles involved and the amount of offending in which juveniles engage. Yet significant heterogeneity exists in offending, with a small proportion of juveniles engaging in high rates of delinquent and criminal behavior over longer periods of time, and a larger group that engages in lower rates for a shorter period of time (Moffitt, 1993).

Advancements in developmental theories (Thornberry, 2004) and statistical modeling techniques have led to a burgeoning of research in the identification of distinct developmental trajectories of delinquency and aggression. Two comprehensive reviews document the breadth and depth of research in this area (Jennings \& Reingle, 2012; Piquero, 2008). In general, studies vary in sample characteristics (e.g., race/ethnicity), composition (e.g., offender vs. non-offender), length between observations, number of observations, and outcome variables (e.g., aggression, police contacts). There are also differences in the number and shapes of trajectory groups across studies. Despite these differences, common patterns have been uncovered, with most studies finding a low-rate group, a high-rate group, and, on average, one to three groups in between characterized by different ages of onset and desistance.

Little attention, however, has been paid to trajectories of delinquency for North American Indigenous adolescents, which limits our understanding of the heterogeneity in aggressive delinquency among Indigenous youth. We could locate only two studies that examined trajectories of offending with Indigenous populations in North America. Yessine and Bonta (2009) compared Indigenous and non-Indigenous juveniles on probation in Canada. For both samples, a chronic-high and stablelow group were identified, although there was a larger (non-significant) proportion of chronic-high offenders in the Indigenous sample. Additionally, the Indigenous chronic high group tended to be first arrested at slightly younger ages than its non-Indigenous counterpart. Recently Reingle and Maldonado-Molina (2012) estimated trajectories of violent behavior and victimization among the Native American subsample in the Add Health study. Three groups were identified: nonviolent, desistors, and escalators; of the three, the nonviolent trajectory was largest $(67 \%)$. Although important, both of these studies are limited in advancing our understanding of trajectories of aggressive behavior and their risk and protective factors among Indigenous youth. Neither study was able to use multivariate analysis because of the small samples used. In the first study, bivariate analysis found few significant risk factors to differentiate the high and low offending groups in the Indigenous sample. The second study did not include risk factors to differentiate between the nonviolent, desisting, or escalating groups, but rather focused on the overlap in violent offending and victimization. National studies such as Add Health that include small numbers of Indigenous youth overlook possible differences between Indigenous tribes and between Indigenous youth living on reservations or in urban areas, which have been documented in substance use research (Beauvais, 1992; Mitchell, Beals, Novins, \& Spicer, 2003).

\section{RISK AND PROTECTIVE FACTORS FOR AGGRESSION}

We draw from the social development model of antisocial behavior (Catalano \& Hawkins, 1996) to provide a theoretically driven rationale for including the proposed risk and protective factors. The model integrates concepts from social bond, social learning, and differential association theories. Youth learn prosocial and antisocial behavior through primary socializing agents such as family, schools, and peers. The model posits two pathways to antisocial behavior via the bonds that are formed through interaction with others and involvement in activities, any of which can be 
either prosocial or antisocial. The development of prosocial bonds influences belief in the moral validity of societal rules and behavior, which is predicted to directly inhibit antisocial behavior. The antisocial pathway suggests that interaction with antisocial individuals and involvement in antisocial behavior promote the formation of antisocial bonds, which have a direct effect on delinquency and an indirect effect through its influence on prodelinquent beliefs. The current study is not a direct test of the social development model but rather uses the model as an organizational and conceptual framework to inform the selection of early risk and protective factors for trajectories of aggressive delinquency, which are reviewed below.

\section{Family, School, and Peer Contexts}

Delinquency research on "parenting" effects has tended to focus on parent-child relationships. Parents are an important influence on adolescent development and the psychological well being of their children, and this influence is expected to differentiate between different trajectories of delinquency (Hoeve et al., 2007). For example, in their study of middle-school students, Griffin et al. (2003) found that parental monitoring reduced delinquency for both boys and girls, and family closeness was associated with less child aggression. Family structure often looks quite different among Indigenous people, with extended family members and elders playing a stronger role in the socialization of youth than is typically seen among nonIndigenous people (Whitbeck, Sittner Hartshorn, \& Walls, 2014); this may provide a key protective factor in reducing or preventing delinquent behavior (Mmari, Blum, \& Teufel-Stone, 2010; Pridemore, 2004).

Just as positive parenting can foster prosocial behavior, poor parenting can increase aggressive behavior and reduce prosocial tendencies. In a metaanalysis by Hoeve et al. (2009), parental rejection was consistently associated with delinquency or aggression, both concurrently and predictively. The authors also pointed out that, across multiple studies, rejection by parents may have stronger effects long-term than short-term, suggesting that juveniles who perceive more rejection by their parents at younger ages may engage in higher levels of delinquency later on. In two studies with the same sample of Indigenous adolescents as in the current study, parental rejection was associated concurrently with bullying behavior (Melander, Sittner Hartshorn, \& Whitbeck, 2013) and with criminal arrest 2 years later (Sittner Hartshorn, Whitbeck, \& Prentice, 2012).

A second set of variables associated with aggressive behavior relates to school. Education is highly valued in Indigenous communities and youth who are academically successful are often given special recognition within their tribes. Yet compared to non-Indigenous youth in the United States and Canada, Indigenous youth are less likely to complete high school and more likely to be held back a grade (Canadian Council on Learning, 2005; Ogunwole, 2006), patterns that have been attributed to more negative experiences in school for Indigenous youth (Crawford, Cheadle, \& Whitbeck, 2010). Doing poorly in school or failing a class is positively associated with aggressive behavior (Vaillancourt, Brittain, McDougall, \& Duku, 2013), although it is also possible that they share common causes (Hinshaw, 1992).

Conversely, school has been identified as a principal contributor to resilience among Indigenous youth (LaFromboise, Hoyt, Oliver, \& Whitbeck, 2006) and may play a particularly important role in protecting against antisocial behavior. For instance, more positive school adjustment reduced the odds of being a bully among a sample of Indigenous adolescents (Melander et al., 2013). Many studies with non-Indigenous youth have found that adolescents who are attached to school, have close ties with teachers, and feel school is important, tend to engage in less undesirable behavior and experience more academic success and motivation (Dornbusch, Erickson, Laird, \& Wong, 2001).

Another key risk factor associated with delinquency is a juvenile's peer group, which can foster either prosocial or antisocial behavior. For Indigenous youth growing up on rural reservations/reserves, peer networks tend to be smaller and more enduring than those found in urban areas, and may exert more influence on behavior when refusal to participate is seen as violating cultural norms of affiliation and respect (Whitbeck et al., 2014). Among other groups of adolescents, associating with delinquent peers has been linked with the onset of delinquency (Elliott \& Menard, 1996).

\section{Other Antisocial Behavior}

American Indian adolescents begin using alcohol and other substances at earlier ages than youth from other ethno-cultural groups (Blum, Harmon, Harris, Bergeisen, \& Resnick, 1992). They are also more likely to be arrested for substance-related offenses (Leonardson, 2006), making substance use a salient correlate for delinquent behavior. Substance use and serious or violent delinquency often co-occur (Huizinga, Loeber, Thornberry, \& Cothern, 2000) and share many of the same predictors (Barnes, Hoffman, Welte, Farrell, \& Dintcheff, 2006). Little research has examined the associations between cigarette smoking and delinquency, although there is some indication that early cigarette smoking is associated with subsequent delinquency (Tucker, Martínez, Ellickson, \& Edelen, 2008). More research has studied alcohol or marijuana and 
aggression. For example, White et al. (1999) showed that frequency of alcohol and marijuana use were significantly correlated with violent behavior in early adolescence, although alcohol exerted a stronger influence on subsequent violence.

Early dating experiences have been shown to increase delinquent and aggressive behavior (Cui, Ueno, Fincham, Donnellan, \& Wickrama, 2012). Although the mechanisms linking adolescent dating with delinquency are not well understood, there may be several reasons why early dating experiences may influence trajectories of aggressive delinquency. First, early dating may be considered an off-time role transition, which can cause strain for adolescents (Cui et al., 2012). Second, early dating predicts later dating behavior. Evidence from the Add Health study indicates that the cumulative number of dating partners increases subsequent delinquency, controlling for prior levels of delinquent behavior (Cui et al., 2012). Third, adolescent dating may exacerbate early risk factors for aggression such as delinquent peer associations and other antisocial behavior (Fidler, West, Jarvis, \& Wardle, 2006). Thus, early dating experiences may be a precursor to a longer term cumulative process linked with trajectories of aggressive behavior. National data from the Youth Behavior Risk Surveillance Survey (Pavkov, Travis, Fox, King, \& Cross, 2010) suggests that Indigenous adolescents are more likely than nonIndigenous adolescents to engage in early (before the age of 14) sexual experiences (a proxy for early dating). Taken together with the extant literature on early dating and delinquency, early dating experiences may be a salient risk factor for aggression trajectories among Indigenous adolescents.

\section{Sex Differences}

There are significant sex differences in delinquency and aggression (Broidy \& Agnew, 1997; Heimer \& De Coster, 1999). In general, boys engage in higher levels than girls, a pattern that appears to be consistent across countries (Broidy et al., 2003). In trajectory studies that include sex as a covariate, girls are more likely to be in the trajectories associated with lower levels of delinquency (Cleverley, Szatmari, Vaillancourt, Boyle, \& Lipman, 2012). For example, in a study of Dutch youth ages 4-18 years, boys had higher levels of aggressive behavior but the trajectories had similar shapes for both sexes (Bongers, Koot, Van Der Ende, \& Verhulst, 2004). Although sex differences are not explicitly part of the social development model, the model has been found to work similarly for boys and girls (Fleming, Catalano, Oxford, \& Harachi, 2002).

The current study uses longitudinal data from 646 Indigenous adolescents from a single culture, spanning the ages of 10-19, to investigate trajectories of aggressive delinquency and their predictors. With the social development model of antisocial behavior as a guiding framework, we predict that having more delinquent peers, failing a class in school, experiencing more parental rejection, and engaging in other antisocial behavior will be associated with higher levels of aggressive behavior across adolescence. We also predict that having more positive relationships with parents and stronger bonds at school will be associated with lower levels of aggressive delinquency.

\section{DATA AND METHODS}

\section{Procedures}

The project was designed in partnership with the participating reservations and reserves. Before the application funding, the research team was invited to work on these reservations, and tribal resolutions were obtained. At each participating location, an advisory board was appointed by the tribal council; these advisory boards were responsible for advising regarding difficult personnel problems, questionnaire development, and ensuring that published reports were respectful and protected the identity of the respondents and the culture. Upon advisory board consensus of the questionnaires, the study procedures and questionnaires were submitted for review by the university institutional review board.

All of the staff on the reservations were either tribal members or, in a very few cases, non-members who are spouses of tribal members who were well known and accepted in the community. Each reservation/reserve had a local Indigenous full-time interviewer supervisor who coordinated visits and provided quality control. The supervisors and advisory boards handled all personnel issues and problems at the local level. After the first year we developed a core of dependable, sensitive, and hardworking interviewers at each site.

To ensure quality of data collection, all the interviewers underwent annual trainings focusing on that year's questionnaire. They included practice interviews and feedback sessions that reviewed interview quality and problem areas such as skip patterns where mistakes were frequently made. In addition, all of the interviewers completed a required human subjects protection training that emphasized the importance of confidentiality and taught procedures to maintain the confidentiality of data. The use of local interviewers in small communities raised concerns about confidentiality and under-reporting. To reduce this, we notified the families in advance who their interview team would be and they had the option to veto one or both of the interviewers and select those with whom they felt more secure. Our feedback was that the local interviewers actually reduced under reporting in that people were so well known to one 
another they were less likely to dissemble in front of the interviewers.

Each of the participating reservations and reserves provided a list of families of tribally enrolled children aged 10-12 years who lived on or proximate (within 50 miles) to the reservation or reserve. We attempted to contact all families with a target child within the specified age range. Families were recruited with a personal visit by an Indigenous interviewer at which time the project was explained to them. They were then presented with a traditional gift and invited to participate. The recruitment method was approved by advisory boards on each of the reservation and reserves. If the family members agreed to be interviewed, each family member received $\$ 40$ for their time when the interviews were completed. The recruitment procedure resulted in an overall response rate of $79.4 \%$.

\section{Sample and Data}

This sample is representative of one the most populous Indigenous cultures in the United States and Canada. The reserves and reservations share a common cultural tradition and language with minor regional variations in dialects. One of the agreements with the participating reservations and reserves was to maintain cultural and community confidentiality, and thus the names of the reservations and reserves will not be provided, nor will the specific Indigenous culture be identified. Instead, and in keeping with research precedents (e.g., the designation of Southwest and Northern Plains cultures in the American Indian Services Utilization, Psychiatric Epidemiology, Risk and Protective Factors Projects (AI-SUPERPFP), we will refer to the culture by its geographical area: the northern Midwest.

These data were collected as part of a longitudinal study on three reservations in the northern Midwest and four Canadian reserves. Data from one reservation is not included because that location did not have an active advisory board. We opted not to include its data out of respect for the community's right to review research prior to publication. Eight waves of data were collected via yearly interviews with the adolescent and at least one primary caretaker; the data used in this study are from the first seven waves. Wave 1 was collected from October 2002 through October 2003; wave 7 was collected from May 2008 to December 2009. Between waves 1 and 7, the sample was reduced from 675 to 570 adolescents, with an overall retention rate of $84.4 \%$. The present study uses data from waves $1,2,3,5$, and 7 (waves 4,6 , and 8 are excluded because only diagnostic data were collected). Only those respondents for whom we had at least three observations for the dependent variable were included in the analyses, which totaled 659 cases or $97.8 \%$ of the sample. Attrition analyses showed no differences in aggressive delinquency between those with at least three and those with fewer observations. Sample characteristics are detailed in Whitbeck et al. (2014).

\section{Measures}

Aggressive delinquency. The dependent variable consists of nine yes/no items assessing aggressive behavior, which were adapted from the conduct disorder module of the Diagnostic Interview Schedule for Child IV (Shaffer, Fisher, Lucas, Dulcan, \& Schwab-Stone, 2000). These items include attacking someone to steal, threatening someone to steal from them, starting a fire without permission, physical cruelty to an animal, bullying others, being in a physical fight, and hurting someone with a weapon $(\alpha=.68-.73)$.

Family, peer, and school context. Two family process variables were examined. First, a measure of perceived parental rejection was created using three items (e.g., how often do you feel that your family blames you even when you don't deserve it). The response options ranged from (0) none of the time to (2) all of the time. The three items were summed to create a scale $(\alpha=.51)$. Second, a measure of perceived positive parenting practices was created using eight items related to monitoring behavior, being warm and supportive, and approving of adolescent behavior (e.g., how often does someone know where you are, how often do you talk to someone in your family about things that bother you). Response options ranged from (1) never to (3) always. All eight items were averaged to create a composite score $(\alpha=.65)$.

Two school-level variables were assessed. First, the respondents were asked whether or not they have ever failed a class in school, coded as one if they said yes. Second, a measure of school adjustment was created with seven items (e.g., you like school a lot, you do well in school). This measure has been previously used and normed for use among Indigenous adolescents (Crawford et al., 2010). Response options for these items were yes and no. The yes responses were summed to create a count of positive school adjustment $(\alpha=.71)$.

A measure of peer delinquency was created using seven questions asking how many of the respondent's three best friends: use tobacco or alcohol, do not get along with their parents, have gotten into trouble at school, have gotten in trouble with the police, are sexually active, and have parents who drink or use drugs. Response options range from (0) no friends to (3) three friends. A composite score was obtained by taking the average of the nine items $(\alpha=.77)$.

Antisocial behavior. Three substance use variables were assessed. Respondents were asked whether they have ever had more than a sip of beer, wine, and/or any other kind of alcoholic beverage. Those who 
responded with a yes to any of these questions were considered to have ever tried alcohol. Respondents were also asked whether or not they have ever smoked cigarettes or marijuana. Those responding with a yes to these questions were considered to have ever tried tobacco and ever tried marijuana, respectively. Early dating was assessed using one dichotomous item which asked whether or not respondents had a steady boyfriend or girlfriend.

Socio-demographic characteristics. We examined low socioeconomic status (SES), which has been associated with both juvenile aggression and delinquency (Wright, Caspi, Moffitt, Miech, \& Silva, 1999). We measured SES using family income based on parent/ caretaker reports to two questions: their combined household income and the number of people living in the household. Total family income was divided by 1,000 and then divided by the number of people living in the household to create a measure of per capita family income. We also examined possible non-linear patterns by splitting the income variable into quartiles to assess whether income only influences aggressive delinquency trajectories at the extremes. We did not observe any clear non-linear patterns for income (results available upon request) and opted to use the continuous measure in all subsequent analyses. To account for sex differences in aggression, described previously, we included adolescent $\operatorname{sex}(1=$ girl, $0=$ boy $)$ as a covariate.

\section{Analytic Strategy}

We used a semi-parametric finite mixture model (Nagin, 1999, 2005) to examine trajectories of aggressive delinquency with seven waves of data using the traj (Jones \& Nagin, 2013) add-on in Stata Version 13. This approach is sometimes referred to as a latent class growth model or group based trajectory model. In general, this statistical model identifies distinct groups of individuals who follow similar developmental trajectory patterns. Because our dependent variable is a count variable with excess zeroes we specified a zero-inflated Poisson distribution.

Models with up to six groups were estimated, and following Nagin's (2005) suggestions, we used a threestep procedure to select the number of groups. First, we used the Bayesian information criteria (BIC) to select the adequate number of groups (Jones, Nagin, \& Roeder, 2001; Raftery, 2005). A five group model resulted in the highest BIC value (one group model: $\mathrm{BIC}=-4,860.60$; two group: $\mathrm{BIC}=-4,204.58$; three group: $\mathrm{BIC}=-4,123.79$; four group: $\mathrm{BIC}=-4,099.35$; five group: $\mathrm{BIC}=-4,065.82$; six group: $\mathrm{BIC}=-4,080.54$ ). Second, to determine the shape of each trajectory group, the polynomial orders for each group were adjusted and the highest BIC value was used to select trajectory

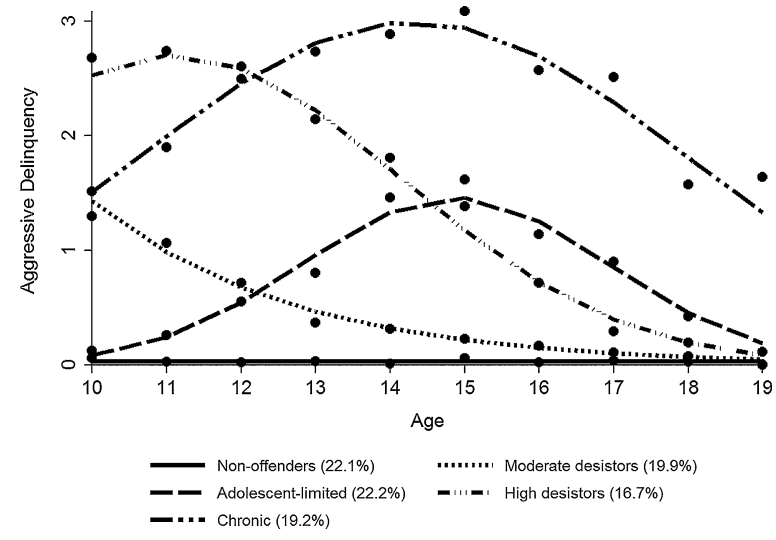

Fig. 1. Group-based trajectories of aggressive delinquency.

shapes (i.e., intercept, linear, quadratic, and cubic). The best fitting model had an intercept term for the first group, a linear term for the second group, and quadratic terms for the last three groups (see Fig. 1).

Third, Nagin (2005) identified multiple diagnostic indicators that can be used to assess model adequacy. We relied on three of these indicators to assess the adequacy of a five group model, as shown in Table I. First, posterior probability values assess the probability of an individual with a certain aggressive delinquency profile belonging to a specific trajectory group. Nagin (2005) suggested that average posterior probability (AvgPP) values of .70 or higher for each group indicates adequate group classification. Each group had an AvgPP value exceeding .70, which suggests that at least $70 \%$ of individuals are correctly classified into their respective trajectory groups. Second, the odds correct classification (OCC) indicator measures the accuracy with which people are assigned to groups. Nagin (2005) suggests that OCC values above 5 for all groups indicate high assignment accuracy. For all groups, OCC values exceeded 5. Third, estimated probabilities of group membership can be compared to the proportion of the sample actually assigned to each group. Values that

TABLE I. Diagnostic Indicators Assessing Model Adequacy

\begin{tabular}{lcccc}
\hline & $\begin{array}{c}\text { Group } \\
\text { Membership } \\
\text { Probability }\end{array}$ & $\begin{array}{c}\text { Proportion } \\
\text { Classified } \\
\text { in Group }^{\mathrm{a}}\end{array}$ & AvgPP & OCC \\
\hline $\begin{array}{l}\text { Non-offenders } \\
\begin{array}{l}\text { Moderate } \\
\quad \text { desistors }\end{array}\end{array}$ & .22 & .25 & .82 & 16.46 \\
$\begin{array}{l}\text { Adolescent- } \\
\quad \text { limited }\end{array}$ & .20 & .20 & .73 & 10.91 \\
$\begin{array}{l}\text { High desistors } \\
\text { Chronic }\end{array}$ & .17 & .21 & .79 & 13.29 \\
\hline
\end{tabular}

AvgPP, average posterior probability; OCC, odds correct classification.

${ }^{\mathrm{a}}$ Based on maximum posterior probability assignment method. 
closely correspond to one another suggest low classification error (Nagin, 2005). The differences in the two values are low for each trajectory group.

Given these criteria, the five group model appears to be a good representation of the data. Compared to models with three and four trajectory groups (results available upon request), the five group model resulted in the highest BIC value, diagnostic criteria indicate high model classification accuracy, and each of the groups has a sizable portion of individuals and makes logical sense. Moreover, the difference in BIC values among the three, four, and five group are substantial enough to warrant selection of a five group model (Raftery, 1995).

In the first set of analyses, the adolescents were categorized into their respective trajectory groups based on their maximum posterior probability of group membership. The distributions (i.e., percentages and means) of the profile variables were examined across aggression trajectory groups. $\chi^{2}$ and ANOVA tests were used to examine, overall, whether the profile variables differed across groups. Based on a significant $\chi^{2}$ or $F$ test, we made post-hoc comparisons using $\chi^{2}$ tests of proportions for categorical profile variables and Tukey's HSD method of multiple comparisons for continuous profile variables.

Moving beyond these simple bivariate analyses, we were particularly interested in examining what early adolescent risk and protective factors predict longer term trajectories of violent behavior. In the second set of analyses, we predicted membership of each aggression trajectory group based on wave 1 risk and protective factors. Because we have more than two trajectory groups, the model reduces to a multinomial logistic regression model with $k-1$ comparison groups. We present the exponentiated coefficients, which can be interpreted as the relative risk of being in a given trajectory group (for a one-unit increase in $x$ ) relative to some reference category. Because group membership classification is probabilistic, group membership is modeled simultaneously with the trajectories to address the possibility of classification error.

\section{RESULTS}

\section{Trajectory Groups}

Five aggression trajectory groups were identified using the above criteria. As Figure 1 shows, there is a group of adolescents who report almost zero aggression across adolescence (non-offenders) and comprise approximately one-fifth of the sample $(22.1 \%)$. There are two groups of adolescents who start off relatively high in aggression and decrease across adolescence. The first group (moderate desistors-19.9\%) started at a moderate level of aggression at age 10 and decreased thereafter. The other group (high desistors-16.7\%) started off high in aggression and showed rapid decreases starting at the age of 12 . There are also two groups of adolescents who started off lower in aggression during early adolescence, increased through mid adolescence, and decreased starting in later adolescence. The first of these groups (adolescent limited-22.2\%) started off very low in aggression, increased and peaked at a moderate level of aggression around the age of 15, and started to decline afterward. The second of these groups (chronic-19.2\%) started off at a moderate level of aggression, increased and peaked at a high level of aggression at approximately 15 years of age, and decreased in later adolescence.

\section{Trajectory Profiles (Bivariate Analyses)}

Table II presents the distributions of the risk and protective factors, and socio-demographic characteristics for each trajectory group. Four out of five of the family, school, and peer variables were significantly different between trajectory groups. Adolescents who reported early family rejection were more likely to be in the chronic group than in the non-offender group $(F=4.65(4,638), P<.01)$. The other three groups were not significantly different from the other trajectory groups. Those who have failed a class in early adolescence were less likely to be in the non-offending group than in the other four groups $\left(\chi^{2}(4)=27.48\right.$, $P<.001)$. Furthermore, those in the chronic group had a significantly higher proportion of adolescents who have ever failed a class than those in the other groups (except for the high desistor group). Adolescents in the chronic and high desistor group had lower mean levels of school adjustment than those in the non-offending and moderate desistor group $(F=9.71(4,640), P<.001)$. Adolescents in the adolescent-limited group were not significantly different from the other four groups. Adolescents in the high desistor and chronic groups had higher mean levels of delinquent peers than the other three groups $(F=15.69(4,638), P<.001)$. The means for positive parenting practices were not significantly different between groups $(F=2.11(4,640), P>.05)$.

All four antisocial behavior risk factors were statistically significant. For the three substance use behavior variables (alcohol, tobacco, and marijuana: $\chi^{2}(4)=47.24,50.36,44.38, P<.001$, respectively), those in the non-offender group were less likely to have tried substances in early adolescence than those in the other four groups. Those in the high desistor and chronic groups were more likely to have tried substances than those in the moderate-level groups. The only exception to this pattern was for marijuana use. Those in the adolescent-limited group were not different from the non-offender group, but were significantly lower than 
TABLE II. Wave 1 Profiles of Violent Delinquency Trajectory Groups and Descriptive Statistics (Means/Percentages)

\begin{tabular}{|c|c|c|c|c|c|c|}
\hline & Non-Offender & Moderate Desistor & Adolescent-Limited & High Desistor & Chronic & Total Sample (SD) \\
\hline \multicolumn{7}{|l|}{ Socio-demographics } \\
\hline Sex $(\operatorname{girl}=1)$ & $66.67 \% \mathrm{a}_{\mathrm{a}}$ & $42.31 \% \%_{\mathrm{bc}}$ & $51.09 \%_{\mathrm{c}}$ & $47.00 \% \mathrm{bc}$ & $36.67 \% \mathrm{~b}$ & $50.29 \%$ \\
\hline Per capita family income & $6.15_{\mathrm{a}}$ & $5.85_{\mathrm{a}}$ & $5.11_{\mathrm{a}}$ & $5.03_{\mathrm{a}}$ & $4.81_{\mathrm{a}}$ & $5.44(4.04)$ \\
\hline Single parent family & $22.64 \% \mathrm{a}$ & $31.54 \% \mathrm{a}$ & $22.63 \% \mathrm{a}$ & $36.00 \% \mathrm{a}$ & $30.83 \% \mathrm{a}$ & $28.19 \%$ \\
\hline \multicolumn{7}{|l|}{ Family, school, and peer context } \\
\hline Parental rejection & $1.99 \mathrm{a}$ & $2.29_{\mathrm{ab}}$ & $2.39_{\mathrm{ab}}$ & $2.35_{\mathrm{ab}}$ & $2.63_{\mathrm{b}}$ & $2.31(1.26)$ \\
\hline Positive parenting & $1.49_{\mathrm{a}}$ & $1.50 \mathrm{a}$ & $1.53_{\mathrm{a}}$ & $1.57_{\mathrm{a}}$ & $1.57_{\mathrm{a}}$ & $1.64(.24)$ \\
\hline Failed a class in school & $13.55 \% \mathrm{a}$ & $24.81 \%_{\mathrm{b}}$ & $25.74 \%_{\mathrm{b}}$ & $33.00 \% \mathrm{bc}$ & $40.34 \%_{\mathrm{c}}$ & $26.09 \%$ \\
\hline Positive school adjustment & $6.42_{\mathrm{a}}$ & $6.12_{\mathrm{a}}$ & $6.00_{\mathrm{ab}}$ & $5.52_{\mathrm{b}}$ & $5.53_{\mathrm{b}}$ & $5.96(1.48)$ \\
\hline Delinquent peer associations & $.43_{\mathrm{a}}$ & $.52_{\mathrm{a}}$ & $.57 \mathrm{a}$ & $.77_{\mathrm{b}}$ & $.90_{\mathrm{b}}$ & $.62(.58)$ \\
\hline \multicolumn{7}{|l|}{ Antisocial behavior } \\
\hline Ever tried alcohol & $3.80 \% \mathrm{a}$ & $11.63 \% \%_{\mathrm{b}}$ & $16.79 \% \%_{\mathrm{b}}$ & $30.00 \%_{\mathrm{c}}$ & $29.41 \%_{\mathrm{c}}$ & $17.59 \%$ \\
\hline Ever tried tobacco & $14.56 \% \mathrm{a}$ & $27.69 \%_{\mathrm{b}}$ & $24.09 \% \mathrm{~b}_{\mathrm{b}}$ & $49.00 \%_{\mathrm{c}}$ & $45.00 \%_{\mathrm{c}}$ & $30.31 \%$ \\
\hline Ever tried marijuana & $2.55 \% \mathrm{a}$ & $9.23 \% \mathrm{~b}$ & $6.57 \% \mathrm{ab}$ & $20.00 \%_{\mathrm{c}}$ & $25.00 \%_{\mathrm{c}}$ & $11.46 \%$ \\
\hline Early dating & $13.25 \% \mathrm{a}$ & $22.40 \%_{\mathrm{b}}$ & $27.41 \%_{\mathrm{bc}}$ & $39.18 \%_{\mathrm{cd}}$ & $46.22 \%_{\mathrm{d}}$ & $28.24 \%$ \\
\hline
\end{tabular}

Note: Means and percentages in the same row that do not share subscripts differ significantly at $P<.05$ using Tukey's HSD method of multiple comparisons for continuous variables and $\chi^{2}$ tests for categorical variables. All test statistics have four degrees of freedom.

the high desistor and chronic groups. Adolescents in the non-offender group were less likely to have been early daters than any of the other groups $\left(\chi^{2}(4)=43.46(4)\right.$, $P<.001)$. Moreover, adolescents in the chronic group were more likely to have been early daters than the two moderate-level groups. Those in the high desistor group were also more likely to date early than those in the moderate desistor group.

Both of the socio-demographic variables examined were statistically significant. Girls were more likely than boys to be in the non-offender group compared to the other four trajectory groups $\left(\chi^{2}(4)=29.70, P<.001\right)$. Girls were also more likely than boys to be in the adolescent-limited group rather than the chronic group. Although the $F$ test for per capita family income was significant $(F=2.68(4,622)$, $P<.05)$, none of the post-hoc comparisons were significantly different from one another.

\section{Predicting Group Membership}

Table III displays the results for the multinomial logistic regression models predicting aggression trajectory group membership. Only those risk and protective factors that were significant in the bivariate analyses were included here. Because the three substance variables behaved similarly across trajectory groups, we decided to create an overall "ever tried substances" variable at wave 1 (any substance use $=1$ ). In the first panel, adolescents in the non-offender group (reference group) are compared to the other four trajectory groups. Compared to the non-offender group, an increase in parental rejection was associated with higher relative risk of being in the chronic group. Of the two school-level predictors, only positive school adjustment was associated with aggression trajectory profile membership. The relative risk of being in the high desistor and chronic groups decreased as positive school adjustment increased, compared to the nonoffender group. The relative risk of being in the high desistor and chronic groups increased as delinquent peer associations increased, compared to the nonoffender group. Never trying substances increased the relative risk of being in the other trajectory groups (except for the adolescent-limited group) than the nonoffender group. Similarly, early dating increased the relative risk of being in the high desistor and chronic groups than in the non-offender group. Girls had a lower relative risk than boys of being in the moderate desistor, high desistor, and chronic groups than in the non-offender group. There were no significant sex differences in membership between the adolescentlimited group and the non-offender group. Per capita family income decreased the relative risk of being in the chronic group than in the non-offender group.

In summary, most of the differences were between the non-offender group, and the high desistor and chronic groups. Higher levels of risk increased the odds of being in these two groups than in the non-offender group, whereas protective factors decreased the risk. Furthermore, there were no significant differences between the adolescent-limited group and the non-offender group on any of the variables.

In the second panel of Table III, we compare the chronic group (reference category) to the moderate desistor, adolescent-limited, and high desistor groups. Of the family, school, and peer variables, only delinquent peer associations was significantly different between groups. As delinquent peer associations increased, the relative risk of being in the moderate 
TABLE III. Multinomial Logistic Regression Models Predicting Group Membership in Aggressive Delinquency Trajectory Groups

\begin{tabular}{|c|c|c|c|c|c|c|c|}
\hline & \multicolumn{4}{|c|}{ Non-Offender Group Compared to } & \multicolumn{3}{|c|}{ Chronic Group Compared to } \\
\hline & $\begin{array}{l}\text { Moderate } \\
\text { Desistor }\end{array}$ & Adolescent-Limited & $\begin{array}{c}\text { High } \\
\text { Desistor }\end{array}$ & Chronic & $\begin{array}{l}\text { Moderate } \\
\text { Desistor }\end{array}$ & Adolescent-Limited & $\begin{array}{l}\text { High } \\
\text { Desistor }\end{array}$ \\
\hline & RRR & RRR & RRR & RRR & RRR & RRR & RRR \\
\hline \multicolumn{8}{|l|}{ Family, school, and peer context } \\
\hline Parental rejection & 1.17 & 1.26 & 1.06 & $1.36^{*}$ & .91 & .99 & .94 \\
\hline Failed a class at school & 1.33 & 1.43 & 1.54 & 2.15 & .65 & .71 & .86 \\
\hline Positive school adjustment & .83 & .84 & $.67^{*}$ & $.65^{*}$ & 1.20 & 1.18 & .97 \\
\hline $\begin{array}{l}\text { Delinquent peer } \\
\text { associations }\end{array}$ & 1.41 & .84 & $2.05^{*}$ & $2.94^{* *}$ & $.48^{*}$ & $.32^{* *}$ & .76 \\
\hline \multicolumn{8}{|l|}{ Antisocial behavior } \\
\hline Ever tried substances & $2.45^{*}$ & .90 & $3.45^{* *}$ & $4.78^{* * *}$ & .68 & $.28^{* *}$ & 2.08 \\
\hline Early dating & 1.30 & 2.31 & $2.76^{*}$ & $2.65^{*}$ & .51 & .77 & .92 \\
\hline \multicolumn{8}{|l|}{ Socio-demographics } \\
\hline $\operatorname{Sex}(\operatorname{girl}=1)$ & $.24^{* * *}$ & .54 & $.41^{*}$ & $.16^{* * *}$ & 1.24 & $2.64^{*}$ & 1.40 \\
\hline Per capita family income & 1.00 & .95 & .96 & $.90^{*}$ & 1.08 & 1.02 & 1.01 \\
\hline
\end{tabular}

desistor and adolescent-limited groups decreased. Only one of the antisocial behavior contrasts was significant. Adolescents who had tried substances had a lower relative risk of being in the adolescent-limited group than in the chronic group. Girls had a higher relative risk of being in the adolescent-limited group than boys. None of the other sex differences were significant.

In summary, there were no significant differences between the chronic and high desistor groups. The only significant contrasts with the other groups suggest that more risk factors decrease the odds of being in the moderate desistor and adolescent-limited groups than in the chronic group. Furthermore, there were few differences among the adolescent-limited, moderate desistor, and high desistor groups (results not shown; available upon request). The only consistent contrast indicated that ever trying a substance increased the relative risk of being in the high desistor group than in the other two groups. Delinquent peer associations also increased the relative risk of being in the high desistor group than in the adolescent-limited group.

\section{DISCUSSION}

There is considerable heterogeneity in the onset, persistence, and predictors of offending during the early life course. Although the body of research on trajectories of offending shows fairly consistent patterns across racial and ethnic groups (for reviews see Jennings \& Reingle, 2012; Piquero, 2008), only two studies have examined trajectories of delinquency with samples of Indigenous youth (Reingle \& Maldonado-Molina, 2012; Yessine \& Bonta, 2009). This is a striking omission in research when we consider that Indigenous youth, particularly those living on reservations and reserves, live and grow in a developmental context unique to Indigenous people in the United States and Canada (Whitbeck et al., 2014). Furthermore, Indigenous youth are over-represented at multiple points in the juvenile justice process and remain at higher risk of criminal justice system involvement in adulthood (Correctional Service of Canada, 2013; Greenfeld \& Smith, 1999). Therefore, it is imperative that we investigate patterns of delinquency for Indigenous youth, including levels or rates of delinquency and changes over time, as well as risk factors that differentiate chronic and less serious offenders.

In the current study, we identified trajectories of aggressive delinquency with a sample of Indigenous youth from the northern Midwest United States and Canada, and used the social development model of antisocial behavior (Catalano \& Hawkins, 1996) as a lens through which to identify and understand risk and protective factors for delinquency. Five trajectory groups of approximately the same size were identified: a non-offending group with low or no aggressive delinquency during adolescence (22.1\%), an adolescent-limited group that increased to a moderate level of delinquency by age 15 and then declined (22.2\%), a group that started at a moderate level but decreased throughout adolescence (19.9\%), a group that started out at a high level of aggressive delinquency but declined after age $11(16.7 \%)$, and a second adolescent-limited 
group that was characterized by a much higher level of aggressive delinquency throughout adolescence $(19.2 \%)$.

These results align with several other delinquency trajectory studies with non-Indigenous samples. In the review by Jennings and Reingle (2012), most studies identified a desistor or low-delinquency group as well as a chronic group, with various groups in between. Our results, however, differ somewhat in the number and shape of trajectories from the only two trajectory analyses with Indigenous samples that we could locate. Yessine and Bonta (2009) found two groups of Indigenous offenders, a low stable and a high increasing group. Because the measure used in that study included violent and nonviolent offending and the sample consisted entirely of probationers, differences between the two studies are likely to be attributable to outcome measures used and sample composition.

The results of the analyses suggest three main conclusions. First, in the multivariate analyses, we found no differences between the non-offender and the adolescent-limited groups. This would appear to support Moffitt's (1993) contention that adolescence-limited youth follow a normative developmental trajectory and are not heavily influenced by early risk factors. It is likely that the small increase in offending during mid adolescence is the result of the time-varying influence of peers that is not captured in this study.

Second, there were no significant differences between the high desistor and the chronic aggression groups. In the subsequent discussion we refer, when appropriate, to the high desistor and chronic aggression groups together as "high aggression trajectories." There is one notable difference between these two groups. The high desistor group shows high levels of aggressive behavior early on and decreases to a very low level thereafter, whereas the chronic group declines by late adolescence, but ends this stage of the life course higher in aggression than the other four groups. The chronic group appears to be entering emerging adulthood at high risk of continued aggressive and possibly other antisocial behavior. Thus, the following discussion on risk and protective factors is highly relevant for understanding possible early prevention and intervention programming that may decrease the odds of cumulative continuity of aggressive behavior and criminal justice system involvement.

Third, the results appear to align with the social development model's conceptualization of prosocial and antisocial pathways (Catalano \& Hawkins, 1996). More specifically, the general pattern of the data suggests that risk factors increase the odds of being in the high aggression trajectories, whereas protective factors decrease the odds of being in these groups. Of the two protective factors we examined, only positive school adjustment was associated with a decrease in the relative risk of being in the two high aggression trajectory groups, than in the non-offender group. There were no significant differences between the trajectory groups for school failure. These findings support prior research among Indigenous youth highlighting schooling as a robust source of resilience (LaFromboise et al., 2006). During early adolescence, schools become an increasingly important socializing agent. Adolescents who develop strong attachments to school and teachers are likely to perceive more, and engage in, opportunities for prosocial involvement and interaction with prosocial others.

Although positive parenting practices did not predict group membership, parental rejection increased the odds of being in the chronic aggression trajectory than in to the non-offender group. These results support a bulk of the literature, which finds strong associations between parental rejection and delinquency (Hoeve et al., 2009). A potential limitation with our parenting measures is that they focus on the primary caretakers. Indigenous cultural groups tend to emphasize extended kinship groups (Whitbeck et al., 2014) that are not necessarily captured by our "family" influence measures. Parental rejection may inhibit the development of strong bonds with a crucial socializing agent, which increases the probability of drifting into delinquent peers groups and engaging in antisocial behavior.

We found that early delinquent peer associations increased the relative risk of being in the two high aggression trajectory groups and the moderate desistor groups than in the non-offender group. Early antisocial influences may increase opportunities to learn prodelinquent beliefs and for actual delinquent behavior (Haynie \& Osgood, 2005). On the reservations/reserves, many of the youth spend their entire childhood and adolescence embedded in the same peer groups (Whitbeck et al., 2014). Consequently, peers are likely to be a salient and less malleable risk factor.

We examined early substance use initiation and early dating as indicators of antisocial behavior. Compared to the non-offender group, ever trying substances increased the relative risk of being in other trajectory groups, except for the adolescent-limited group. This finding is notable because Indigenous adolescents tend to initiate substance use earlier than other racial/ethnic groups (Bachman et al., 1991). Delaying or preventing the onset of substance use has the potential to reduce aggression among North American Indigenous youth. In addition to substance use initiation, adolescents who were early daters were more likely to be in the two high aggression trajectory groups than the non-offender group. Prior research suggests that early dating is linked with greater delinquent peer involvement (Fidler et al., 2006), and that Indigenous youth engage in earlier sexual behavior 
(a proxy for early dating) than non-Indigenous youth (Pavkov et al., 2010). As such, early substance use and dating behavior may increase the perception and availability of antisocial attachments and opportunities to engage in aggressive behavior.

We included adolescent sex as a predictor of trajectory group membership. Girls were more likely than boys to be in the non-offender and adolescent-limited groups. Our findings are consistent with other trajectory studies that showed girls more likely to be in the low delinquency trajectories (Bongers et al., 2004; Cleverley et al., 2012).

\section{Limitations}

There are several limitations. First, these data come from a single Indigenous culture that is located on rural reservations and reserves. There is considerable heterogeneity across Indigenous groups, with 564 federally recognized tribes in the United States and 615 First Nations communities in Canada. We should be cautious in generalizing the results of this study to adolescents of other culture groups or even to adolescents from the same culture group living in urban areas. Indigenous adolescents living in urban areas develop in very different social and economic contexts, including exposure to different values and cultural groups. For example, in Mmari et al.'s (2010) qualitative study of three different tribal communities, the youth from the urban location were exposed to more violence, including gangs and weapons in school than rural reservation youth. The risk and protective factors identified in the current study may be less relevant for urban youth.

Second, we note some limitations regarding the predictor variables, which are drawn from the baseline wave. They are measured concurrently with the first time point of the aggressive delinquency trajectories and consequently the observed associations may be inflated. Additionally, the baseline risk and protective factors do not capture dynamic processes that might influence behavior. Future research should incorporate time-varying variables to identify age-graded risk and protective factors that might affect movement into and out of trajectory groups. For example, psychosocial risk factors at a later age might better explain the increase in aggressive delinquency for the adolescent-limited group and set it apart from the nonoffender group, which the baseline variables could not do. It may also be beneficial to examine whether there are distinct profiles of risk and protective factors, which might condition the aggressive delinquency trajectories.

The variables in the current study represent major social domains in which adolescents interact, but it would be fruitful for future research to examine a broader array of risk and protective factors that might further differentiate the groups. For example, discrimination has been associated with a host of negative outcomes for youth and adults (Williams \& Mohammed, 2009), including aggressive behavior for Indigenous youth (Sittner Hartshorn, Whitbeck, \& Hoyt, 2012). Experiences with discrimination may differentially effect youth, with those following the chronic trajectory being more vulnerable to its effects given that they already experience more risk factors and fewer protective factors. It is important to examine how and to what extent discrimination contributes to the continuity of aggressive behavior.

Third, our dependent variable is a count that captures the diversity of aggressive acts in which youth might engage, but does not reflect the frequency with which they commit those acts. We are unable to determine, for instance, how often in the past year an adolescent engaged in fights. The number of aggressive acts decreased from three at age 14 to about one by age 19 for the chronic group, which demonstrates that the types of behavior decrease in number but tells us nothing about the frequency with which they occurred. This may be why, unlike other trajectory studies (e.g., Nagin et al., 2003), we did not identify a group that continued at a high level of aggressive behavior, a group often referred to as life-course persistent (Moffitt, 1993). Yet ours is hardly an unusual finding as other trajectory studies also found a group that exhibited a similar pattern of behavior (Hoeve et al., 2007; Lacourse, Nagin, Tremblay, Vitaro, \& Claes, 2003). It is possible that our chronic group will persist in some form of aggressive behavior into adulthood, just not multiple types of behavior, for this group continued to have higher levels of aggression than all of the other groups at age 19.

\section{CONCLUSION}

The results provide important information that could be used in developing prevention and intervention programs, particularly regarding vulnerable ages as well as malleable risk factors. Identifying those youth most at risk of greater involvement in delinquency over longer periods of time may be key to preventing delinquency and reducing the over-representation of Indigenous youth in the juvenile and criminal justice systems. Aggressive delinquency is but one of many related forms of negative behavior in which some adolescents engage, and programs that target multiple types of behavior are likely to be more efficacious and long lasting. In addition, risk and protective factors are distributed across multiple domains of development. As such, prevention and intervention programming must aim to decrease risk factors and promote protective factors within family, school, peer, and individual domains. Most importantly, however, these programs must pay close attention to the unique developmental context in 
which Indigenous adolescents are embedded. Although many of the risk and protective factors are similar to those found in general studies, the social and historical forces (e.g., historical cultural losses) that have shaped and continue to influence these risk and protective factors must be conceptualized and incorporated into prevention programming (Whitbeck et al., 2014).

\section{ACKNOWLEDGMENTS}

The authors would like to thank Les Whitbeck, the tribal advisory boards, and the anonymous reviewers for their helpful comments on this article.

\section{REFERENCES}

Bachman, R. (1992). Death and violence on the reservation: Homicide, family violence, and suicide in American Indian populations. New York: Auburn House.

Barnes, G. M., Hoffman, J. H., Welte, J. W., Farrell, M. P., \& Dintcheff, B. A. (2006). Effects of parental monitoring and peer deviance on substance use and delinquency. Journal of Marriage and Family, 68, 1084-1104. doi: 10.1111/j.1741-3737.2006.00315.x

Beauvais, F. (1992). Comparison of drug use rates for reservation Indian, non-reservation Indian and Anglo youth. American Indian and Alaska Native Mental Health Research, 5, 13-31.

Blum, R. W., Harmon, B., Harris, L., Bergeisen, L., \& Resnick, M. D. (1992). American Indian-Alaska Native youth health. Journal of the American Medical Association, 267, 1637-1644. doi: 10.1001/ jama.1992.03480120075036

Blumstein, A., Cohen, J., \& Farrington, D. P. (1988). Criminal career research: Its value for criminology. Criminology, 26, 1-35. doi: 10.1111/j.1745-9125.1988.tb00829.x

Bongers, I. L., Koot, H. M., van Der Ende, J., \& Verhulst, F. C. (2004). Developmental trajectories of externalizing behaviors in childhood and adolescence. Child Development, 75, 1523-1537. doi: 10.1111/ j.1467-8624.2004.00755.x

Broidy, L., \& Agnew, R. (1997). Gender and crime: A general strain theory perspective. Journal of Research in Crime and Delinquency, 34, 275-306. doi: 10.1177/0022427897034003001

Broidy, L. M., Nagin, D. S., Tremblay, R. E., Bates, J. E., Brame, B., Dodge, K. A., ... Vitaro, F. (2003). Developmental trajectories of childhood disruptive behaviors and adolescent delinquency: A sixsite, cross-national study. Developmental Psychology, 39, 222-245. doi: 10.1037/0012-1649.39.2.222

Canadian Council on Learning. (2005). Good news: Canada's high school dropout rates are falling. Lessons in Learning. Retrieved from http:// www.ccl-cca.ca/pdfs/LessonsInLearning/Dec-16-05-Good-News.pdf

Catalano, R. F., \& Hawkins, J. D. (1996). The social development model: A theory of antisocial behavior. In J. D. Hawkins (Ed.), Delinquency and crime: Current theories (pp. 28-67). New York, NY: Cambridge University Press.

Centers for Disease Control and Prevention. (2013). Youth risk behavior system. Retrieved from http://www.cdc.gov/HealthyYouth/yrbs/index.htm.

Cleverley, K., Szatmari, P., Vaillancourt, T., Boyle, M., \& Lipman, E. (2012). Developmental trajectories of physical and indirect aggression from late childhood to adolescence: Sex differences and outcomes in emerging adulthood. Journal of the American Academy of Child \& Adolescent Psychiatry, 51, 1037-1051. doi: 10.1016/j.jaac.2012.07.010

Correctional Service of Canada. (2013). Deomographic overview of Aboriginal peoples in Canada and Aboriginal offenders in federal corrections. Retrieved from http://www.csc-scc.gc.ca/aboriginal/ 002003-1008-eng.shtml

Crawford, D. M., Cheadle, J. E., \& Whitbeck, L. B. (2010). Tribal vs. public schools: Perceived discrimination and school adjustment among Indigenous children from early to mid-adolescence. Journal of American Indian Education, 49, 80-100.

Cui, M., Ueno, K., Fincham, F. D., Donnellan, M. B., \& Wickrama, K. A. S. (2012). The association between romantic relationships and delinquency in adolescence and young adulthood. Personal Relationships, 19, 354-366. doi: 10.1111/j.1475-6811.2011.01366.x

DeLisi, M., Hochstetler, A., Johnson, G. J., Caudill, J. W., \& Marquart, J. W. (2011). The road to murder: The enduring criminogenic effects of juvenile confinement among a sample of adult career criminals. Youth Violence and Juvenile Justice, 9, 207-221. doi: 10.1177/1541204010396107

Dornbusch, S. M., Erickson, K. G., Laird, J., \& Wong, C. A. (2001). The relation of family and school attachment to adolescent deviance in diverse groups and communities. Journal of Adolescent Research, 16, 396-422. doi: 10.1177/0743558401164006

Duran, E., \& Duran, B. (1995). Native American postcolonial psychology. Albany, NY: State University of New York Press.

Elliott, D. S., \& Menard, S. (1996). Delinquent friends and delinquent behavior: Temporal and developmental patterns. In J. D. Hawkins (Ed.), Delinquency and crime: Current theories (pp. 28-67). Cambridge, UK: Cambridge University Press.

Fleming, C. B., Catalano, R. F., Oxford, M. L., \& Harachi, T. W. (2002). A test of generalizability of the social development model across gender and income groups with longitudinal data from the elementary school developmental period. Journal of Quantitative Criminology, 18, 423-437. doi: 10.1023/A:1021173711746

Fidler, J. A., West, R., Jarvis, M. J., \& Wardle, J. (2006). Early dating predicts smoking during adolescence: A prospective study. Addiction, 101, 1805-1813. doi: 10.1111/j.1360-0443.2006.01613.x

Greenfeld, L. A., \& Smith, S. K. (1999). American Indians and crime (No NCJ 173386). Washington, DC: Bureau of Justice Statistics.

Griffin, K. W., Botvin, G. J., Scheier, L. M., Doyle, M. M., \& Williams, C. (2003). Common predictors of cigarette smoking, alcohol use, aggression, and delinquency among inner-city minority youth. Addictive Behaviors, 28, 1141-1148. doi: 10.1016/S0306-4603(02) 00225-3

Haynie, D. L., \& Osgood, D. W. (2005). Reconsidering peers and delinquency: How do peers matter? Social Forces, 84, 1109-1130. doi: 10.1353/sof.2006.0018

Hinshaw, S. P. (1992). Externalizing behavior problems and academic underachievement in childhood and adolescence: Causal relationships and underlying mechanisms. Psychological Bulletin, 111, 127-155. doi: 10.1037/0033-2909.111.1.127

Heimer, K., \& De Coster, S. (1999). The gendering of violent delinquency. Criminology, 37, 277-317. doi: 10.1111/j.1745-9125.1999.tb00487.x

Hoeve, M., Blokland, A., Dubas, J. S., Loeber, R., Gerris, J. R. M., \& Laan, P. H. (2007). Trajectories of delinquency and parenting styles. Journal of Abnormal Child Psychology, 36, 223-235. doi: 10.1007/s10802-007-9172-x

Hoeve, M., Dubas, J. S., Eichelsheim, V. I., van der Laan, P. H., Smeenk, W., \& Gerris, J. R. M. (2009). The relationship between parenting and delinquency: A meta-analysis. Journal of Abnormal Child Psychology, 37, 749-775. doi: 10.1007/s10802-009-9310-8

Huizinga, D., Loeber, R., Thornberry, T. P., \& Cothern, L. (2000). Cooccurrence of delinquency and other problem behaviors (No. NCJ 182211) (pp. 1-7). Washington, DC: U. S. Department of Justice.

Jennings, W. G., \& Reingle, J. M. (2012). On the number and shape of developmental/life-course violence, aggression, and delinquency trajectories: A state-of-the-art review. Journal of Criminal Justice, 40, 472-489. doi: 10.1016/j.jcrimjus.2012.07.001 
Jones, B. L., \& Nagin, D. S. (2013). A note on a Stata plugin for estimating group-based trajectory models. Sociological Methods \& Research, 42, 608-613. doi: 10.1177/0049124113503141

Jones, B. L., Nagin, D. S., \& Roeder, K. (2001). A SAS procedure based on mixture models for estimating developmental trajectories. Sociological Methods \& Research, 29, 374-393. doi: 10.1177/0049124101029003005

Lacourse, E., Nagin, D., Tremblay, R. E., Vitaro, F., \& Claes, M. (2003). Developmental trajectories of boys' delinquent group membership and facilitation of violent behaviors during adolescence. Development and Psychopathology, 15, 183-197. doi: 10.1017/s0954579403000105

LaFromboise, T. D., Hoyt, D. R., Oliver, L., \& Whitbeck, L. B. (2006). Family, community, and school influences on resilience among American Indian adolescents in the upper Midwest. Journal of Community Psychology, 34, 193-209. doi: 10.1002/jcop.20090

Leonardson, G. R. (2006). Native American crime in the Northwest: 2004-2005-BIA information from Alaska, Montana, Wyoming, Idaho, Oregon, and Washington. Dillon, MT: Montana Board of Crime Control.

Loeber, R., Farrington, D. P., \& Waschbusch, D. A. (1998). Serious and violent juvenile offenders. In R. Loeber \& D. P. Farrington (Eds.), Serious and violent juvenile offenders: Risk factors and successful interventions (pp. 13-29). Thousand Oaks, CA: Sage Publications.

Melander, L. A., Sittner Hartshorn, K. J., \& Whitbeck, L. B. (2013). Correlates of bullying behaviors among a sample of North American Indigenous adolescents. Journal of Adolescence, 36, 675-684. doi: 10.1016/j.adolescence.2013.05.003

Mitchell, C. M., Beals, J., Novins, D. K., \& Spicer, P. (2003). Drug use among two American Indian populations: Prevalence of lifetime use and DSM-IV substance use disorders. Drug and Alcohol Dependence, 69, 29-41. doi: 10.1016/s0376-8716(02) 00253-3

Mmari, K. N., Blum, R. W., \& Teufel-Stone, N. (2010). What increases risk and protection for delinquent behaviors among American Indian youth?: Findings from three tribal communities. Youth \& Society, 41, 382-413. doi: 10.1177/0044118x09333645

Moffitt, T. E. (1993). Adolescence-limited and life-course-persistent antisocial behavior: A developmental taxonomy. Psychological Review, 100, 674-701. doi: 10.1037/0033-295X.100.4.674

Nagin, D. S. (1999). Analyzing developmental trajectories: A semiparametric, group-based approach. Psychological Methods, 4, 139-157. doi: 10.1037/1082-989X.4.2.139

Nagin, D. S. (2005). Group-based modeling of development. Cambridge, MA: Harvard University Press.

Nagin, D. S., Pagani, L., Tremblay, R. E., \& Vitaro, F. (2003). Life course turning points: The effect of grade retention on physical aggression. Development and Psychopathology, 15, 343-361. doi: 10.1017/ S0954579403000191

Ogunwole, S. U. (2006). We the people: American Indians and Alaska Natives in the United States. U.S. Census Bureau: U.S. Department of Commerce.

Pavkov, T. W., Travis, L., Fox, K. A., King, C. B., \& Cross, T. L. (2010). Tribal youth victimization and delinquency: Analysis of youth risk behavior surveillance survey data. Cultural Diversity and Ethnic Minority Psychology, 16, 123-134. doi: 10.1037/a0018664

Perry, S. W. (2004). American Indians and crime-A BJS statistical profile, 1992-2002 (No. NCJ 203097) (pp. 1-56). U.S. Department of Justice: Bureau of Justice Statistics.

Piquero, A. R. (2008). Taking Stock of developmental trajectories of criminal activity over the life course. In A. M. Liberman (Ed.), The long view of crime: A synthesis of longitudinal research (pp. 23-78). New York: Springer.

Pridemore, W. A. (2004). Review of the literature on risk and protective factors of offending among Native Americans. Journal of Ethnicity in Criminal Justice, 2, 45-63. doi: 10.1300/J222v02n04_03
Raftery, A. E. (1995). Bayesian model selection in social research. Sociological Methodology, 25, 111-164. doi: 10.2307/271063

Reingle, J. M., \& Maldonado-Molina, M. M. (2012). Victimization and violent offending: An assessment of the victim-offender overlap among Native American adolescents and young adults. International Criminal Justice Review, 22, 123-138. doi: 10.1177/1057567712443966

Royal Commission on Aboriginal Peoples. (1996). Highlights from the report of the Royal Commission on Aboriginal Peoples. Retrieved July 2, 2010, from http://www.ainc-inac.gc.ca/ap/pubs/rpt/rpt-eng.asp

Sedlak, A. J., \& Bruce, C. (2010). Youth's characteristics and backgrounds: Findings from the survey of youth in residential placement. Juvenile Justice Bulletin. Washington, D.C.: Office of Juvenile Justice and Delinquency Prevention.

Shaffer, D., Fisher, P., Lucas, C. P., Dulcan, M. K., \& Schwab-Stone, M. E. (2000). NIMH diagnostic interview schedule for children version iv (NIMH DISC-IV): Description, differences from previous versions, and reliability of some common diagnoses. Journal of the American Academy of Child and Adolescent Psychiatry, 39, 28-38. doi: 10.1097/ 00004583-200001000-00014

Sittner Hartshorn, K. J., Whitbeck, L. B., \& Hoyt, D. R. (2012). Exploring the relationships of perceived discrimination, anger, and aggression among North American Indigenous adolescents. Society and Mental Health, 2, 53-67. doi: 10.1177/2156869312441185

Sittner Hartshorn, K. J., Whitbeck, L. B., \& Prentice, P. (2012). Substance use disorders, comorbidity, and arrest among Indigenous adolescents. Crime \& Delinquency. Advance online publication. doi: $10.1177 / 0011128712466372$

Statistics Canada. (2001). A profile of criminal victimization: Results of the 1999 general social survey. Ottawa, Ontario: Canadian Centre for Justice Statistics.

Sweeten, G. (2006). Who will graduate? Disruption of high school education by arrest and court involvement. Justice Quarterly, 23, 462-480. doi: 10.1080/07418820600985313

Thornberry, T. P. (Ed.). (2004). Developmental theories of crime and delinquency (Vol. 7). New Brunswick, NJ: Transaction Publishers.

Tucker, J. S., Martínez, J. F., Ellickson, P. L., \& Edelen, M. O. (2008). Temporal associations of cigarette smoking with social influences, academic performance, and delinquency: A four-wave longitudinal study from ages 13-23. Psychology of Addictive Behaviors, 22, 1-11. doi: 10.1037/0893-164X.22.1.1

Vaillancourt, T., Brittain, H. L., McDougall, P., \& Duku, E. (2013). Longitudinal links between childhood peer victimization, internalizing and externalizing problems, and academic functioning: Developmental cascades. Journal of Abnormal Child Psychology, 41, 1203-1215. doi: 10.1007/s10802-013-9781-5

Whitbeck, L. B., Sittner Hartshorn, K. J., \& Walls, M. (2014). Indigenous adolescent development: Psychological, social and historical contexts. New York, NY: Routledge.

White, H. R., Loeber, R., Stouthamer-Loeber, M., \& Farrington, D. P. (1999). Developmental associations between substance use and violence. Development and Psychopathology, 11, 785-803. doi: 10.1017/S0954579499002321

Williams, D. R., \& Mohammed, S. A. (2009). Discrimination and racial disparities in health: Evidence and needed research. Journal of Behavioral Medicine, 32, 20-47. doi: 10.1007/s10865-008-9185-0

Wright, B. R. E., Caspi, A., Moffitt, T. E., Miech, R. A., \& Silva, P. A. (1999). Reconsidering the relationship between SES and delinquency: Causation but not correlation. Criminology, 37, 175-194. doi: 10.1111/j.1745-9125.1999.tb00483.x

Yessine, A. K., \& Bonta, J. (2009). The offending trajectories of youthful Aboriginal offenders. Canadian Journal of Criminology and Criminal Justice, 51, 435-472. doi: 10.3138/cjccj.51.4.435 